\title{
Assessment of best single sample for finding chlamydia in women with and without symptoms: a diagnostic test study
}

\author{
(c) $\frac{(1)(8)}{\text { gy }}$ OPEN ACCESS
}

\section{Sarah A Schoeman consultant in genitourinary medicine ${ }^{1}$, Catherine M W Stewart specialty registrar in genitourinary medicine ${ }^{1}$, Russell A Booth lead biomedical scientist ${ }^{2}$, Susan D Smith advanced biomedical scientist and training coordinator ${ }^{2}$, Mark $\mathrm{H}$ Wilcox professor of clinical microbiology ${ }^{2}$, Janet $\mathrm{D}$ Wilson consultant in genitourinary medicine ${ }^{1}$}

'Department of Genitourinary Medicine, Leeds General Infirmary, Leeds LS1 3EX, UK; ${ }^{2}$ Department of Clinical Microbiology, Leeds General Infirmary

\begin{abstract}
Objective To compare vulvovaginal swabs with endocervical swabs as optimal diagnostic sample for detection of Chlamydia trachomatis infection.

Design A diagnostic test study.

Setting An urban sexual health centre.

Participants 3973 women aged $\geq 16$ years requesting testing for sexually transmitted infections.

Interventions Participants took a vulvovaginal swab before routine examination, and clinicians took an endocervical swab during examination
\end{abstract}

Main outcome measure Diagnosis of chlamydia infection with samples analysed using the Aptima Combo-2 assay; positive results confirmed with the Aptima CT assay.

Results Of the 3973 participants, 410 (10.3\%) were infected with $C$ trachomatis. Infected women were significantly younger (22 v 25 years, $\mathrm{P}<0.0001)$ and more likely to have symptoms suggestive of a bacterial sexually transmitted infection $(53 \%$ v $41 \%$, odds ratio $1.63(95 \% \mathrm{Cl} 1.30$ to 2.04$)$ ), be a contact of someone with a sexually transmitted infection (25\% v5\%, odds ratio 6.18 (4.61 to 8.30$)$ ), clinically diagnosed with cervicitis (17\% v4\%, odds ratio 4.92 (3.50 to 6.91)), and have pelvic inflammatory disease ( $9 \% \vee 3 \%$, odds ratio 2.85 (1.87 to 4.33$)$ ). When women co-infected with gonorrhoea were included in the analysis, there was an association with mixed ethnicity $(10 \% v 7 \%$, odds ratio $1.53(1.07$ to 2.17)); but when those with gonorrhoea were removed, women of white ethnicity were significantly more likely to have chlamydia ( $85 \% \mathrm{~V}$ $80 \%$, odds ratio 1.40 (1.03 to 1.91$)$ ). On analysis of complete paired results, vulvovaginal swabs were significantly more sensitive than endocervical swabs (97\% (95\% Cl $95 \%$ to $98 \%$ ) v $88 \%(85 \%$ to $91 \%)$, $\mathrm{P}<0.00001$ ); corresponding specificities were $99.9 \%$ and $100 \%$. In women with symptoms suggestive of a bacterial sexually transmitted infection, vulvovaginal swabs were significantly more sensitive than endocervical swabs $(97 \%$ (93\% to $98 \%$ ) v $88 \%$ ( $83 \%$ to $92 \%), \mathrm{P}=0.0008)$, as they were in women without symptoms (97\% (94\% to $99 \%) v 89 \%(84 \%$ to 93\%), $P=0.002$ ).

Conclusions Vulvovaginal swabs are significantly better than endocervical swabs at detecting chlamydia in women with and without symptoms suggestive of sexually transmitted infections. In those with symptoms, using endocervical samples rather than vulvovaginal swabs would have missed $9 \%$ of infections, or 1 in every 11 cases of chlamydia.

Trial registration ISRCTN42867448.

\section{Introduction}

Chlamydia is the most common sexually transmitted infection diagnosed and treated in the United Kingdom, ${ }^{1}$ with the highest rates in young people under the age of 25 years. It is often asymptomatic but can have serious sequelae if left untreated. In women, ascending infection causes pelvic inflammatory disease with potential complications of infertility and ectopic pregnancy. Chlamydia trachomatis can infect the urethra or endocervix, or both. Studies looking at sites of chlamydia infection in women found chlamydia present in both urethra and endocervix in $73-76 \%$, endocervix only in $15-16 \%$, and urethra only in $10-11 \% .{ }^{23}$ The optimal diagnostic sample must be able to detect the maximum number of infected people. Also, as up to $70 \%$ of infected females can be asymptomatic, it is important that diagnostic tests have good sensitivity and specificity at detecting chlamydia in women both with and without symptoms.

Nucleic acid amplification tests are the "gold standard" for $C$ trachomatis detection because of their high sensitivity and specificity. With these tests, numerous studies have shown that non-invasively obtained samples are as accurate as those 
obtained from the urethra and endocervix. ${ }^{4}$ For women without symptoms, non-invasive samples eliminate some of the barriers to screening for chlamydia as they require no examination and are clearly preferred by patients. ${ }^{56}$ The non-invasive samples used in women are first-catch urine samples or self collected vulvovaginal swabs. Evidence suggests that self collected vulvovaginal swabs have the better sensitivity, probably because they collect more material from the two potential sites of infection..$^{7-12}$

The Gen-Probe Aptima Combo-2 assay uses transcription mediated amplification and automatically tests for both chlamydia and gonorrhoea on each sample at no extra cost compared with testing for one pathogen. However, only one swab can be included in each assay tube. Before switching to Aptima Combo-2, our practice in testing women who needed an examination because they had symptoms had been to place swabs from both the urethra and endocervix in the same transport medium for analysis by our previous assay. On transferring to the Aptima Combo-2 assay, we needed to choose between endocervical and vulvovaginal sampling. Most studies have shown that the two samples are equivalent, ${ }^{4} 10$ 13-19 suggesting that, when a woman is undergoing an examination, an endocervical swab would be preferable on the assumption that it is superior for detecting low chlamydial load in the endocervix (which if missed could leave women at risk of ascending infection). However, one study found vulvovaginal swabs to be significantly more sensitive than endocervical swabs $(81 \% v 65 \%)$, although the authors commented that the sensitivity of the endocervical swabs was lower than in previously published studies. ${ }^{9}$ Hence, it remains unclear which is the optimum sample in women requiring examination.

As part of a study comparing self taken vulvovaginal swabs with clinician taken urethral and endocervical swabs for the detection of gonorrhoea, ${ }^{20}$ we were able to evaluate the diagnostic accuracy of self taken vulvovaginal swabs versus clinician taken endocervical swabs for the detection of chlamydia, using the Aptima Combo-2 assay.

\section{Methods}

The full methods for the study are described elsewhere. ${ }^{20}$ Women aged $\geq 16$ years who presented to the Centre for Sexual Health at Leeds for a new visit were invited to participate. Exclusion criteria were having taken antibiotics in the preceding 28 days and being unable or unwilling to take a vulvovaginal swab or to have the standard examination and swabs performed by a clinician. We collected details of the women's age, ethnicity, history of sexually transmitted infections, and being in contact with a sexually transmitted infection. During the medical history, symptoms in keeping with a bacterial sexually transmitted infection (vaginal discharge, dysuria, intermenstrual or postcoital bleeding, deep dyspareunia, and lower abdominal pain) were recorded. During the examination the presence of cervicitis and pain or tenderness on bimanual pelvic examination in keeping with a clinical diagnosis of pelvic inflammatory disease were noted.

The self taken vulvovaginal swab was taken before examination. The women were then examined, and the clinician took an endocervical sample. Consequently, each woman had samples for chlamydia taken from two different sites, the vulvovagina and endocervix, and these were both analysed for $C$ trachomatis by means of the Aptima Combo-2 assay. The assays were performed at the Department of Microbiology by accredited laboratory staff. The vulvovaginal swabs and endocervical samples were assayed according to the manufacturer's instructions (Gen-Probe, San Diego CA, USA). The assay employs transcription mediated amplification technology in which rRNA target molecules from $C$ trachomatis are isolated and specific regions are amplified by using a separate capture oligomer and a unique set of primers.

The cut-off values for the chlamydia results were determined by Tigris software for each run. The results of the initial Aptima Combo-2 assay were therefore positive, equivocal, or negative for $C$ trachomatis. Positive or equivocal tests were further analysed using the Aptima CT mono-specific platform assay, which has a different target to the Aptima Combo-2. The samples were only reported as clinically positive if confirmed as positive by the Aptima CT. This algorithm of repeat testing for confirmation is currently the suggested national standard operating procedure in England. ${ }^{21}$ A positive or equivocal Aptima Combo-2 assay unconfirmed by Aptima CT was reported as indeterminate. A patient was considered to have $C$ trachomatis infection if an endocervical or vulvovaginal swab gave at least one positive Aptima Combo-2 test result that was confirmed by the Aptima CT mono-specific platform test. As the cut-off values for the chlamydia results were determined by the Tigris software for each run, the results of these could not be influenced by the laboratory staff.

\section{Statistical analysis}

We used the Mann Whitney U test to determine any associations between the presence of $C$ trachomatis and age and the $\chi^{2}$ test to determine associations between $C$ trachomatis and the categorical variables. The overall sensitivity, specificity, positive predictive value, and negative predictive value were calculated for the Aptima Combo-2 assay with an endocervical swab and the Aptima Combo-2 assay with a vulvovaginal swab. These analyses were also performed for women with and without symptoms. Any differences between sensitivities were compared using McNamar's test on paired samples.

\section{Results \\ Participants}

Full demographic data and infection status were available for 3973 women recruited by 42 different clinicians, both doctors and nurses, between March 2009 and January 2010. The participants' mean age was 25 years (range 16-59), and self reported ethnicity was $80 \%$ white $(n=3171), 9 \%$ black $(362)$, $7 \%$ mixed (297), and $4 \%$ other (143). A previous diagnosis of sexually transmitted infection was reported in 1478 (37\%), and 292 (7\%) reported contact with a partner recently diagnosed with a sexually transmitted infection. At least one symptom suggestive of a bacterial sexually transmitted infection was reported by 1671 (42\%) of the participants. A clinical diagnosis of cervicitis was made in $218(5 \%)$, and $169(4 \%)$ had a clinical diagnosis of pelvic inflammatory disease.

In all, 410 of the 3973 women had $C$ trachomatis infection (prevalence 10.3\%). The factors associated with having chlamydia are shown in table $1 \Downarrow$. Fifty five $(13.4 \%)$ of the women with chlamydia were co-infected with Neisseria gonorrhoeae, leaving 355 women with chlamydia but no gonorrhoea. In total, 100 of the 3973 women were infected with gonorrhoea (prevalence $2.5 \%$ ), leaving 3518 women (88.5\%) who were chlamydia and gonorrhoea negative. As gonorrhoea is more likely to cause symptoms and signs suggestive of a bacterial sexually transmitted infection, the data have been analysed including and excluding the women infected with gonorrhoea (table $1 \Downarrow$ ). 
In the whole cohort, women with chlamydia, were significantly more likely to be of mixed ethnicity, to be younger, to have symptoms suggestive of a bacterial sexually transmitted infection, to be a contact of a sexually transmitted infection, and to be clinically diagnosed as having cervicitis and pelvic inflammatory disease, irrespective of whether they were co-infected with gonorrhoea. However, when those infected with gonorrhoea are removed, women of white ethnicity were significantly more likely to have chlamydia compared with non-white women. Women of "other" ethnic origin were significantly less likely to have chlamydia compared with white women irrespective of whether they were co-infected with gonorrhoea.

\section{Test sensitivities and specificities}

Some test results were missing because of problems with sample collection: 29 of the endocervical swabs taken by clinicians could not be processed because of staff errors in labelling, and 77 of the self taken vulvovaginal swabs could not be processed because of participant errors with the sample tube or staff errors in labelling. This meant that $106(2.7 \%)$ of the women recruited had to be removed before statistical analysis with the paired McNemar's test. Table $2 \Downarrow$ shows the test results for the 3867 women with complete paired results. Of these, $396(10.2 \%)$ were positive for chlamydia. The sensitivities of the clinician taken endocervical swabs and self taken vulvovaginal swabs were $88 \%$ and $97 \%$ respectively $(\mathrm{P}<0.00001)$.

Only $10(0.13 \%)$ of the 7734 chlamydia samples were reported as indeterminate, six vulvovaginal swabs and four endocervical swabs. Of the six indeterminate vulvovaginal swab results, three were positive with the Aptima Combo- 2 assay but unconfirmed by the Aptima CT assay. Two of these were classified as false positives as the respective endocervical results were negative, and one was classified as a true positive as the respective endocervical result was positive. The remaining three indeterminate vulvovaginal swab results were equivocal with Aptima Combo-2 and unconfirmed with Aptima CT. Two of these had negative endocervical results and so were classified as true negatives, and the other had a positive endocervical result and was classified as a false negative. Of the four endocervical swabs that gave indeterminate results, two had positive Aptima Combo- 2 results that were unconfirmed by Aptima CT. As the respective vulvovaginal swabs were positive, they were classified as true positives. The other two had equivocal Aptima Combo-2 results that were unconfirmed by Aptima CT. As the vulvovaginal swabs were negative, they were classified as true negatives. Table $3 \Downarrow$ shows the sensitivities, specificities, positive predictive values, and negative predictive values for the Aptima Combo- 2 assay.

\section{Diagnostic accuracy of tests in women with and without symptoms}

At least one symptom suggestive of a bacterial sexually transmitted infection was reported by $1634(42 \%)$ of the participants with complete paired results. Of these, 213 (13\%) women were infected with chlamydia. The sensitivities of clinician taken endocervical swabs and self taken vulvovaginal swabs were $88 \%(187 / 213)$ and $97 \%$ (206/213) respectively $(\mathrm{P}=0.0008)$.

There were 2233 (58\%) women who did not have symptoms suggestive of a bacterial sexually transmitted infection, 183 (8\%) of whom were infected with chlamydia. The sensitivities of clinician taken endocervical swabs and self taken vulvovaginal swabs were 89\% (163/183) and 97\% (178/183) respectively $(\mathrm{P}=0.0025)$.

There were 1347 women (35\%) who would have been suitable for an "asymptomatic screen"- that is, they did not have any symptoms suggestive of a bacterial sexually transmitted infection and did not require examination for any other reason (such as symptoms of genital skin pathology). Of these, 76 $(5.6 \%)$ had chlamydia infection. The sensitivities of clinician taken endocervical swabs and self taken vulvovaginal swabs were $89 \%$ (68/76) and 95\% (72/76) respectively $(\mathrm{P}=0.27)$.

\section{Discussion}

This is the first study to show that vulvovaginal swabs analysed by Aptima Combo-2 are significantly better at detecting $C$ trachomatis than endocervical swabs in women with and without symptoms of a sexually transmitted infection. The overall sensitivities were $97 \%$ for vulvovaginal swabs and $88 \%$ for endocervical swabs, meaning that endocervical swabs diagnosed $9 \%$ fewer infections, missing one in every 11 cases of chlamydia. One previous study also found vulvovaginal swabs to be significantly more sensitive than endocervical swabs (at $81 \%$ versus $65 \%$ respectively), but the authors commented that the sensitivity of the endocervical swabs was lower than in previously published studies, which may have been due to a different laboratory being used for their analysis. ${ }^{9}$ Some other studies have reported higher (but non-significant) chlamydia sensitivities with vulvovaginal swabs compared with endocervical swabs.

We have identified 11 published papers where there are comparisons between vulvovaginal swabs and endocervical swabs using nucleic acid amplification tests for the detection of chlamydia. ${ }^{7-11}$ 13-19 $^{-}$One included a comparison using two different nucleic acid amplification tests, making 12 comparisons in total. ${ }^{19}$ Two of the comparisons found a higher sensitivity with the endocervical swabs, with differences of $1.8 \%{ }^{15}$ and $0.6 \%,{ }^{11}$ whereas 10 found a higher sensitivity with the vulvovaginal swabs, with a median of $5.1 \%$ difference and a range of 1.2-16.0\%..$^{710131416-19}$ Exact data were available in nine of the 11 papers..$^{9-11}{ }^{13-18}$ Combining the results, the endocervical swabs diagnosed chlamydia in 772/892 (86.5\%) and the vulvovaginal swabs diagnosed chlamydia in $824 / 886$ (93.0\%), a difference of $6.5 \%$. As reported in other studies, we found excellent specificity using both the endocervical swabs $(100 \%)$ and the vulvovaginal swabs $(99.9 \%)$ for the detection of chlamydia with the Aptima Combo-2 assay. ${ }^{192}$

We think the difference in sensitivity between endocervical swabs and vulvovaginal swabs is probably due to missed urethral infections when the endocervical site alone is sampled. This theory is supported by a study that investigated use of using first-catch urine samples in addition to endocervical swabs; it found that endocervical swabs tested by Aptima Combo-2 detected $99.4 \%$ of cervical $C$ trachomatis infections but only $92.1 \%$ of the total $C$ trachomatis infections because some were detected only with first-catch urine. This reduction in sensitivity was greater when testing for chlamydia than for gonorrhoea. ${ }^{22}$ Also, a study comparing sensitivities of endocervical swabs, vulvovaginal swabs, and first-catch urine samples, separately or in combination, for the detection of chlamydia found the separate endocervical swab, vulvovaginal swab, and first-catch urine samples detected $65 \%, 81 \%$, and $72 \%$ respectively, whereas the endocervical swab plus first-catch urine detected $86 \%$, the endocervical and vulvovaginal swabs detected $91 \%$, and the vulvovaginal swab plus first-catch urine detected $94 \%$. 


\section{Implications of study results}

The higher chlamydia diagnostic sensitivity with vulvovaginal swabs in our study was seen in all women irrespective of whether they had symptoms suggestive of a bacterial sexually transmitted infection. In those who would not routinely require a genital examination, endocervical swabs and vulvovaginal swabs were equivalent. Our study therefore confirms the previous evidence that in women without symptoms, and for those who do not require a speculum examination, the sample of choice is a self taken vulvovaginal swab, which is an appropriate sample to test for both chlamydia and gonorrhoea. ${ }^{20}$ In women with symptoms the sensitivity of chlamydia detection by means of vulvovaginal swabs was significantly higher than for endocervical swabs (97\% versus $88 \%$ ). Using endocervical samples rather than vulvovaginal swabs would have missed $9 \%$ of infections, or one in every 11 cases of chlamydia. These findings give a clear indication that the best single sample for chlamydia detection in women with symptoms is the vulvovaginal swab. Therefore, in women with symptoms, where a speculum examination would be normal practice, we recommend either a self taken vulvovaginal swab before the examination or a clinician taken vulvovaginal swab before the speculum insertion. These have been shown to be equivalent in sensitivity, ${ }^{10}$ but the clinician taken sample has the advantage that patient errors in collection are eliminated.

The associations of chlamydia infection with younger age and mixed ethnicity, and decreased likelihood in women of "other" ethnicity are in keeping with data from the National Chlamydia Screening Programme (NCSP), which found that chlamydia disproportionately affects young adults and black or mixed ethnic groups. ${ }^{23}$ However, some women with chlamydia (13.4\% in our study) are co-infected with gonorrhoea, which is also associated with black or mixed ethnicity. ${ }^{20}$ Interestingly when those with gonorrhoea were removed from our data, white women were significantly more likely to have chlamydia compared with non-white women. Our findings suggest that when assessing any association between ethnicity and chlamydia infection, gonorrhoea is a confounding factor. The NCSP data do not identify those with and without gonorrhoea, as chlamydia status is tested but not necessarily gonorrhoea status. From our study findings, we suggest white ethnicity should be considered as a risk factor for chlamydia infection in the UK.

\section{Strengths and limitations of study}

The strengths of our study include its large number of participants with 42 different clinicians collecting the samples-as such, it is reflective of real clinical situations. The population attending the Centre for Sexual Health at Leeds is similar to many other clinic populations, both in the UK and in other countries, meaning our findings are widely applicable.

The limitations are that, although there were a large number of participants, this was a single centre study. The order of the samples was not randomised or rotated. The self taken vulvovaginal swab was collected before the clinician taken endocervical swab. In other published studies comparing vulvovaginal swabs with endocervical samples, the vulvovaginal swab was performed first, before the insertion of a vaginal speculum. ${ }^{79^{10} 1^{16-19}}$ As the sites for these samples are different, there is no reason to suspect that taking the vulvovaginal swab would affect the sensitivity of the endocervical sample.

However, as part of the diagnostic comparisons for gonorrhoea in this study, ${ }^{20}$ all participants had an endocervical swab taken for gonorrhoea culture, which was performed before the endocervical swab for Aptima Combo-2 assay in view of the reduced sensitivity of culture.

It could be argued that taking the endocervical culture sample first reduces the sensitivity of the endocervical sample for nucleic acid amplification tests, which might account for the lower sensitivity in our endocervical samples compared with the vulvovaginal swabs. We believe this is unlikely, as a comparison of endocervical swabs and cytobrushes (using a less sensitive test than a nucleic acid amplification test) showed no difference in the rate of detection of $C$ trachomatis irrespective of the order in which they were performed. ${ }^{24}$

Further limitations are that negative Aptima Combo-2 tests were not repeated, so we could have missed some false negative results. This is unlikely, however, as each participant had two different samples analysed for chlamydia. As we assessed only one nucleic acid amplification test, our results cannot necessarily be extrapolated to other such tests. The sensitivity and specificity of different nucleic acid amplification tests for chlamydia detection can vary, as reported in a systematic review. ${ }^{4}$

\section{Conclusions}

The optimal diagnostic sample must be able to detect the maximum number of infected people, and we found that vulvovaginal swabs analysed by Aptima Combo-2 for chlamydia infection were significantly more sensitive than endocervical swabs, which missed $9 \%$ of cases in routine clinical practice. Therefore, in women who do not require a speculum examination the sample of choice is a self taken vulvovaginal swab. This has benefits for the patient in that a genital examination is avoided, and it has health economic benefits in that clinician time and equipment use is reduced. For women with symptoms, we would recommend either a self taken vulvovaginal swab before the examination or a clinician taken vulvovaginal swab before speculum insertion.

Contributors: JDW conceived the study and wrote the protocol with assistance from MHW. CMWS, SAS, and JDW recruited participants, and RAB, SDS, and MHW performed the microbiological testing. CMWS and SAS coordinated the study and with JDW produced the database and analysed the data. All authors contributed to writing the paper and had full access to all of the data in the study and can take responsibility for the integrity of the data and the accuracy of the data analysis. JDW and MHW are the guarantors for the study.

Funding: No specific funding for the study.

Competing interests: All authors have completed the ICMJE uniform disclosure form at www.icmje.org/coi_disclosure.pdf (available on request from the corresponding author) and declare: extra diagnostic reagents and equipment needed for the study were provided by Gen-Probe; no financial relationships with any organisations that might have an interest in the submitted work in the previous three years; no other relationships or activities that could appear to have influenced the submitted work.

Ethical approval: Leeds (East) Research Ethics Committee granted ethical approval for the study. All participants gave informed consent before taking part in the study.

Data sharing: No additional data available

Health Protection Agency. Sexually transmitted infections in England. Health Protection Report 2011;5(24). www.hpa.org.uk/hpr/archives/2011/hpr2411.pdf.

2 Buimer M, van Doornum GJJ, Ching S, Peerbooms PG, Plier PK, Ram D, et al. Detection of Chlamydia trachomatis and Neisseria gonorrhoeae by ligase chain reaction-based assays with clinical specimens from various sites: implications for diagnostic testing and screening. J Clin Microbiol 1996;34:2395-400.

3 Hay PE, Thomas BJ, Horner PJ, MacLeod E, Renton AM, Taylor-Robinson D. Chlamydia trachomatis in women: the more you look, the more you find. Genitourin Med 1994;70:97-100. 


\section{What is already known on this topic}

The optimal diagnostic sample for Chlamydia trachomatis detection should detect the maximum number of infected people

In women without symptoms the sample of choice is a self taken vulvovaginal swab, but it is not clear whether a vulvovaginal swab or an endocervical swab is the optimum sample in women with symptoms requiring speculum examination

\section{What this study adds}

In this study of women attending a sexual health centre vulvovaginal swabs were significantly better at detecting chlamydia infection than endocervical swabs among women with symptoms of a sexually transmitted infection

In these cases, using endocervical samples rather than vulvovaginal swabs would have missed $9 \%$ of infections, or 1 in 11 cases of chlamydia infection

Women with symptoms should have either a self taken vulvovaginal swab before examination or a clinician taken vulvovaginal swab before speculum insertion

4 Cook RL, Hutchison SL, Ostergaard L, Braithwaite RS, Ness RB. Systematic review: Non-invasive testing for Chlamydia trachomatis and Neisseria gonorrhoeae. Ann Intern Med 2005:142:914-25.

5 Wiesenfeld HC, Lowry DL, Heine RP, Krohn MA, Bittner H, Kellinger K, et al. Self-collection of vaginal swabs for the detection of chlamydia, gonorrhoea, and trichomoniasis: opportunity to encourage sexually transmitted disease testing among adolescents. Sex Transm Dis 2001;28:321-5.

6 Chernesky MA, Hook EW III, Martin DH, Lane J, Johnson R, Jordan JA, et al. Women find it easy and prefer to collect their own vaginal swabs to diagnose Chlamydia trachomatis or Neisseria gonorrhoeae infections. Sex Transm Dis 2005;32:729-33.

7 Schachter J, McCormack WM, Chernesky MA, Martin DH, Van Der Pol B, Rice PA, et al. Vaginal swabs are appropriate specimens for diagnosis of genital tract infection with Chlamydia trachomatis. J Clin Microbiol 2003;41:3784-9.

8 Skidmore S, Horner P, Herring A, Sell J, Paul I, Thomas J, et al. Vulvovaginal swab or first-catch urine specimen to detect Chlamydia trachomatis in women in a community setting? J Clin Microbiol 2006;44:4389-94.

9 Shafer MA, Moncada J, Boyer CB, Betsinger K, Flinn SD, Schachter J. Comparing first-void urine specimens, self collected vaginal swabs, and endocervical specimens to detect Chlamydia trachomatis and Neisseria gonorrhoeae by a nucleic acid amplification test. $J$ Clin Microbiol 2003;41:4395-9.

10 Schachter J, Chernesky MA, Willis DE, Fine PM, Martin DH, Fuller D, et al. Vaginal swabs are the specimens of choice when screening for Chlamydia trachomatis and Neisseria gonorrhoeae: results from a multicenter evaluation of the APTIMA assays for both infections. Sex Transm Dis 2005;32:725-8.

11 Falk L, Coble B-I, Mjornberg P-A, Fredlund H. Sampling for Chlamydia trachomatis infection-a comparison of vaginal, first-catch urine, combined vaginal and first-catch urine and endocervical sampling. Int J STD AIDS 2010;21:283-7.

12 National Chlamydia Screening Steering Group. New frontiers: annual report of the National Chlamydia Screening Programme in England 2005/6. Health Protection Agency, 2006.

13 Stary A, Schuh E, Kerschbaumer M, Götz B, Lee H. Performance of transcription-medicated amplification and ligase chain reaction assays for detection of chlamydial Infection in urogential samples obtained by invasive and noninvasive methods. J Clin Microbiol 1998;36:2666-70.

14 Garrow SC, Smith DW, Harnett GB. The diagnosis of chlamydia, gonorrhoea, and trichomonas infections by self obtained low vaginal swabs, in remote northern Australian clinical practice. Sex Transm Infect 2002;78:278-81.

15 O'Neil D, Doseeva V, Rothmann T, Wolff J, Nazarenko I. Evaluation of Chlamydia trachomatis and Neisseria gonorrhoeae detection in urine, endocervical, and vaginal specimens by a multiplexed isothermal thermophilic helicase-dependent amplification (tHDA) assay. J Clin Microbiol 2011:49:4121-5.
16 Fang J, Husman C, DeSilva L, Chang R, Peralta L. Evaluation of self-collected vaginal swab, first void urine, and endocervical swab specimens for the detection of Chlamydia trachomatis and Neisseria gonorrhoeae in adolescent females. J Pediatr Adolesc Gynecol 2008;21:355-60.

17 Blake DR, Maldeis N, Barnes MR, Hardick A, Quinn TC, Gaydos CA. Cost-effectiveness of screening strategies for Chlamydia trachomatis using cervical swabs, urine, and self-obtained vaginal swabs in a sexually transmitted disease clinic setting. Sex Transm Dis 2008;35:649-55.

18 Taylor SN, Van Der Pol B, Lillis R, Hook EW 3rd, Lebar W, Davis T, et al. Clinical evaluation of the BD ProbeTecTM Chlamydia trachomatis $Q x$ amplified DNA assay on the BD ViperTM system with XTRTM technology. Sex Transm Dis 2011;38:603-9.

19 Gaydos CA, Cartwright CP, Colaninno P, Welsch J, Holden J, Ho SY, et al. Performance of the Abbott RealTime CT/NG for detection of Chlamydia trachomatis and Neisseria gonorrhoeae. J Clin Microbiol 2010;48:3236-43.

20 Stewart CMW, Schoeman SA, Booth RA, Smith SD, Wilcox MH, Wilson JD. Assessment of self taken swabs versus clinician taken swab cultures for diagnosing gonorrhoea in women: single centre, diagnostic accuracy study. BMJ 2012;345:e8107.

21 Health Protection Agency. National standard method: chlamydia infection-testing by nucleic acid amplification tests VSOP. HPA, 2005

22 Moncada J, Schachter J, Hook EW III, Ferrero D, Gaydos C, Quinn TC, et al. The effect of urine testing in evaluations of the sensitivity of the Gen-Probe APTIMA Combo 2 assay on endocervical swabs for Chlamydia trachomatis and Neisseria gonorrhoeae. Sex Transm Dis 2004;31:273-7.

23 Simms I, Talebi A, Rhia J, Horner P, French RS, Sarah R, et al. The English National Chlamydia Screening Programme: variations in positivity in 2007/2008. Sex Transm Dis 2009;36;522-7

24 Kellogg JA, Seiple JW, Klinedinst JL, Levisky JS. Comparison of cytobrushes with swabs for the recovery of endocervical cells and for Chlamydiazyme detection of Chlamydia trachomatis. J Clin Microbiol 1992;30:2988-90.

\section{Accepted: 13 November 2012}

\section{Cite this as: BMJ 2012;345:e8013}

This is an open-access article distributed under the terms of the Creative Commons Attribution Non-commercial License, which permits use, distribution, and reproduction in any medium, provided the original work is properly cited, the use is non commercial and is otherwise in compliance with the license. See: http://creativecommons.org/licenses/by$\mathrm{nc} / 2.0 /$ and http://creativecommons.org/licenses/by-nc/2.0/legalcode. 


\section{Tables}

Table 1| Factors associated with Chlamydia trachomatis infection among 3973 women recruited at a sexual health centre. Values are numbers (percentages) of women unless stated otherwise

Positive for chlamydia Negative for chlamydia $(n=410) \quad(n=3563)$
Chlamydia prevalence $(\%) \quad$ Odds ratio $(95 \% \mathrm{Cl})$ for infection ${ }^{\star} \quad$ P value $^{\star}$

Including women infected with Neisseria gonorrhoeae $(\mathrm{n}=3973)$

\begin{tabular}{|c|c|c|c|c|c|}
\hline Mean age (years) & 22 & 25 & 10.3 & & $<0.0001$ \\
\hline Symptoms of STI & $218(53)$ & $1453(41)$ & 13.0 & 1.65 (1.34 to 2.03$)$ & $<0.0001$ \\
\hline Previous STI & $151(37)$ & $1327(37)$ & 10.2 & 0.98 (0.79 to 1.22$)$ & 0.91 \\
\hline Contact of person with STI & $104(25)$ & $188(5)$ & 35.6 & 6.10 (4.63 to 8.03$)$ & $<0.0001$ \\
\hline Cervicitis & $76(19)$ & $142(4)$ & 34.9 & 5.48 (4.01 to 7.48$)$ & $<0.0001$ \\
\hline Pelvic inflammatory disease & $40(10)$ & $129(4)$ & 23.7 & 2.88 (1.95 to 4.23$)$ & $<0.0001$ \\
\hline \multicolumn{6}{|l|}{ Ethnicity: } \\
\hline White & $335(82)$ & $2836(80)$ & 10.6 & $1.15(0.87$ to 1.50$)$ & 0.35 \\
\hline Black & $30(7)$ & $332(9)$ & 8.3 & 0.77 (0.51 to 1.15$)$ & 0.21 \\
\hline Mixed & $43(10)$ & $254(7)$ & 14.5 & 1.53 (1.07 to 2.17$)$ & 0.018 \\
\hline Other & $2(<1)$ & $141(4)$ & 1.4 & $0.12(0.02$ to 0.49$)$ & 0.0006 \\
\hline
\end{tabular}

Excluding women infected with Neisseria gonorrhoeae $(\mathrm{n}=3873)$

\begin{tabular}{|c|c|c|c|c|c|}
\hline No of women & 355 & 3518 & & & \\
\hline Mean age (years) & 22 & 25 & 9.2 & & $<0.0001$ \\
\hline Symptoms of STI & $187(53)$ & $1427(41)$ & 11.6 & $1.63(1.30$ to 2.04$)$ & $<0.0001$ \\
\hline Previous STI & $130(37)$ & $1302(37)$ & 9.1 & 0.99 (0.78 to 1.25$)$ & 0.98 \\
\hline Contact of person with STI & $88(25)$ & $178(5)$ & 33.1 & $6.18(4.61$ to 8.30$)$ & $<0.0001$ \\
\hline Cervicitis & $59(17)$ & $137(4)$ & 30.1 & 4.92 (3.50 to 6.91$)$ & $<0.0001$ \\
\hline Pelvic inflammatory disease & $33(9)$ & $122(3)$ & 21.3 & 2.85 (1.87 to 4.33$)$ & $<0.0001$ \\
\hline \multicolumn{6}{|l|}{ Ethnicity†: } \\
\hline White & $301(85)$ & $2812(80)$ & 9.7 & $1.40(1.03$ to 1.91$)$ & 0.03 \\
\hline Black & $25(7)$ & $322(9)$ & 7.2 & $0.73(0.46$ to 1.13$)$ & 0.16 \\
\hline Mixed & $27(7)$ & $244(7)$ & 10.0 & $1.03(0.67$ to 1.59$)$ & 0.96 \\
\hline Other & $2(<1)$ & $140(4)$ & 1.4 & $0.13(0.02$ to 0.54$)$ & 0.002 \\
\hline
\end{tabular}

STI=sexually transmitted infection.

*Mean ages compared by Mann-Whitney U test, all other factors compared by Pearson's $X^{2}$ test with Yates correction.

†White women compared with non-white groups combined. Non-white groups compared with white women. 
Table 2| Comparison of self taken vulvovaginal swabs with clinician taken endocervical swabs as diagnostic samples for detection of Chlamydia trachomatis infection among 3867 women recruited at a sexual health centre. Values are numbers of women unless stated otherwise

\begin{tabular}{|c|c|c|c|c|c|}
\hline & \multicolumn{2}{|c|}{ Chlamydia infection status } & \multirow[b]{2}{*}{ Total } & \multirow[b]{2}{*}{ \% Sensitivity (95\% Cl) } & \multirow[b]{2}{*}{ P value* } \\
\hline & Positive & Negative & & & \\
\hline \multicolumn{6}{|c|}{ Whole cohort } \\
\hline \multicolumn{4}{|c|}{ Endocervical swab: } & 88 (85 to 91$)$ & $<0.00001$ \\
\hline Positive & 350 & 0 & 350 & & \\
\hline Negative & 46 & 3471 & 3517 & & \\
\hline Total & 396 & 3471 & 3867 & & \\
\hline \multicolumn{4}{|c|}{ Vulvovaginal swab: } & 97 (95 to 98 ) & \\
\hline Positive & 384 & 2 & 386 & & \\
\hline Negative & 12 & 3469 & 3481 & & \\
\hline Total & 396 & 3471 & 3867 & & \\
\hline
\end{tabular}

Women with symptoms suggestive of bacterial sexually transmitted infection

\begin{tabular}{|c|c|c|c|c|c|}
\hline \multicolumn{4}{|c|}{ Endocervical swab: } & \multirow[t]{4}{*}{88 (83 to 92$)$} & \multirow[t]{8}{*}{0.0008} \\
\hline Positive & 187 & 0 & 187 & & \\
\hline Negative & 26 & 1421 & 1447 & & \\
\hline Total & 213 & 1421 & 1634 & & \\
\hline \multicolumn{4}{|c|}{ Vulvovaginal swab: } & \multirow[t]{4}{*}{97 (93 to 98$)$} & \\
\hline Positive & 206 & 1 & 207 & & \\
\hline Negative & 7 & 1420 & 1427 & & \\
\hline Total & 213 & 1421 & 1634 & & \\
\hline \multicolumn{6}{|c|}{ Women without symptoms suggestive of bacterial sexually transmitted infection } \\
\hline \multicolumn{4}{|c|}{ Endocervical swab: } & \multirow[t]{4}{*}{89 (84 to 93$)$} & \multirow[t]{8}{*}{0.0025} \\
\hline Positive & 163 & 0 & 163 & & \\
\hline Negative & 20 & 2050 & 2070 & & \\
\hline Total & 183 & 2050 & 2233 & & \\
\hline \multicolumn{4}{|c|}{ Vulvovaginal swab: } & \multirow[t]{4}{*}{97 (94 to 99) } & \\
\hline Positive & 178 & 1 & 179 & & \\
\hline Negative & 5 & 2049 & 2054 & & \\
\hline Total & 183 & 2050 & 2233 & & \\
\hline \multicolumn{6}{|c|}{ Women suitable for asymptomatic screen } \\
\hline \multicolumn{4}{|c|}{ Endocervical swab: } & \multirow[t]{4}{*}{89 (81 to 95$)$} & \multirow[t]{8}{*}{0.2668} \\
\hline Positive & 68 & 0 & 68 & & \\
\hline Negative & 8 & 1271 & 1279 & & \\
\hline Total & 76 & 1271 & 1347 & & \\
\hline \multicolumn{4}{|c|}{ Vulvovaginal swab: } & \multirow[t]{4}{*}{95 (87 to 98$)$} & \\
\hline Positive & 72 & 1 & 73 & & \\
\hline Negative & 4 & 1270 & 1274 & & \\
\hline Total & 76 & 1271 & 1347 & & \\
\hline
\end{tabular}

*Endocervical swab compared with vulvovaginal swab. 
Table 3/ Sensitivities, specificities, and positive and negative predictive values of testing for Chlamydia trachomatis infection with self taken vulvovaginal swabs or with clinician taken endocervical swabs as diagnostic samples among 3867 women recruited at a sexual health centre. Values are percentages

\begin{tabular}{|c|c|c|c|c|}
\hline & \multirow[b]{2}{*}{ Sensitivity (95\% Cl) } & \multirow[b]{2}{*}{ Specificity $(95 \% \mathrm{Cl})$} & \multicolumn{2}{|c|}{ Predictive value } \\
\hline & & & Positive & Negative \\
\hline \multicolumn{5}{|l|}{ Whole cohort } \\
\hline Endocervical swab & 88 (85 to 91$)$ & 100 (99.9 to 100$)$ & 100 & 98.7 \\
\hline Vulvovaginal swab & 97 (95 to 98 ) & 99.9 (99.8 to 100$)$ & 99.5 & 99.7 \\
\hline \multicolumn{5}{|c|}{ Women with symptoms suggestive of bacterial sexually transmitted infection } \\
\hline Endocervical swab & 88 (83 to 92$)$ & 100 (99.7 to 100$)$ & 100 & 98.2 \\
\hline Vulvovaginal swab & 97 (93 to 98 ) & 99.9 (99.6 to 100$)$ & 99.5 & 99.5 \\
\hline \multicolumn{5}{|c|}{ Women without symptoms suggestive of bacterial sexually transmitted infection } \\
\hline Endocervical swab & 89 (84 to 93$)$ & 100 (99.8 to 100$)$ & 100 & 99.0 \\
\hline Vulvovaginal swab & 97 (94 to 99) & 99.9 (99.7 to 100$)$ & 99.4 & 99.8 \\
\hline \multicolumn{5}{|c|}{ Women suitable for asymptomatic screen } \\
\hline Endocervical swab & 89 (81 to 95$)$ & 100 (99.7 to 100$)$ & 100 & 99.4 \\
\hline Vulvovaginal swab & 95 (87 to 98$)$ & 99.9 (99.6 to 100$)$ & 98.6 & 99.7 \\
\hline
\end{tabular}

